\title{
AN EIGHT YEAR STUDY OF THE RADIO EMISSION FROM THE WR BINARY HD 193793
}

\author{
ROBERT H. BECKER \\ Physics Dept., University of California, Davis, CA 95616, USA \\ and Lawrence Livermore National Laboratory/IGPP \\ and \\ RICHARD L. WHITE \\ Space Telescope Science Institute, 3700 San Martin Dr., Baltimore, MD 21218, USA
}

\begin{abstract}
Although we now know of quite a few WR-O star binary systems, only HD 193793 is well studied across the entire electromagnetic spectrum. Hence it affords us the best opportunity to test various models for the system against a wealth of observational data. In this paper we present the results of 8 years of monitoring the radio flux density from HD 193793 with the VLA. This database is unique both in terms of its dense coverage of an entire binary cycle and because it extends the radio coverage to $2 \mathrm{~cm}$ wavelength, a shorter wavelength than previously available. With this data we are able to simultaneously solve for the time dependent attenuation in the system and the intrinsic radio luminosity. The standard model of spherically symmetric colliding winds faces severe difficulties in explaining the observations. We conclude that the radio data are most readily interpreted in the context of a WR star wind which is confined to a disk. A disk model for the WR wind also provides a natural explanation for the sudden formation of dust just after periastron.
\end{abstract}

\section{Introduction}

A significant number of WR stars are known to reside in binary systems with $\mathrm{O}$ star companions. At least some of these systems have highly elliptical orbits $(e=0.8)$. Generally speaking, in these systems both stars exhibit mass loss which in turn results in a variety of observable phenomena. The most interesting of these arise from the interaction between the WR and $\mathrm{O}$ star winds. These phenomena can vary wildly over a binary cycle as the interaction zone moves closer to or further from the two stars.

Perhaps the best studied of these systems is HD 193793 (WR140). In 1987, Williams et al. (1987) concluded that HD 193793's two stars (WC6 and 06; McDonald 1947) had a 7.9 year binary orbit based on two dust formation episodes which occurred near periastron (= phase 0$)$. The orbit is highly elliptical, with an eccentricity of 0.84 (Williams et al. 1990), see Fig. 1). From a number of observations it was clear that the radio emission from HD 193793 also varied dramatically over the binary period (Florkowski 1982 , Becker \& White 1985). At radio quiescence near phase 0.1 , the emission appears consistent with the thermal free-free emission from the WR star's ionized wind (which presumably dominates by a large factor over the $\mathrm{O}$ star wind). Later in the orbit the radio emission increases markedly and changes character, becoming primarily non-thermal. This non-thermal emission is generally accepted as arising from the interaction zone of the two winds. 


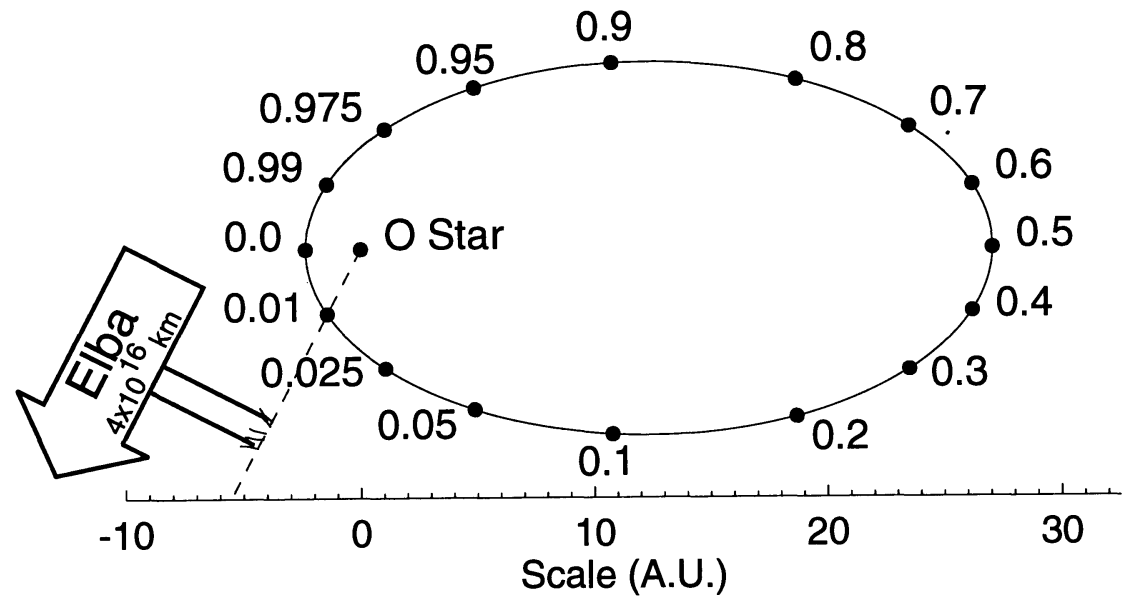

Fig. 1. Orbit of HD 193793. The $\mathrm{O}$ star is located at the origin; the position of the WR star as a function of phase is marked, as is the viewing direction.

Considerable work has been centered on interpreting the phase dependence of the non-thermal radio emission. As early as 1984 it became apparent to us that the solution of this problem required a more detailed radio lightcurve. Starting in 1985 we began to monitor HD 193793 with the NRAO Very Large Array (VLA) and have continued to do so through August 1993. In this paper, we present the results of the VLA monitoring program and use the observed light-curve to constrain the geometry of the WR wind and its interaction with the $\mathrm{O}$ star wind.

\section{Observations}

Approximately once a month for the past eight years beginning in October 1985, 1.5-2 hours of VLA time have been allocated to observe HD 193793. Most of the observations were made at two wavelengths $(2$ and $6 \mathrm{~cm})$ except for the time interval October 1990 to October 1992 when $20 \mathrm{~cm}$ was observed as well. The observations were made in standard continuum mode with $50 \mathrm{MHz}$ bandpasses on two IFs. The observations were calibrated against either $3 \mathrm{C} 48$ or $3 \mathrm{C} 286$ as well as a nearby secondary calibrator. By necessity, the observations were made in all VLA configurations resulting in a rather nonuniform data set. However, since HD 193793's non-thermal emission is unresolved by the VLA even in the A configuration, the measured flux densities should be configuration-independent except in quiescence. The data were calibrated, self-calibrated, and imaged using standard AIPS routines. The resulting light-curves at 2, 6, and $20 \mathrm{~cm}$ are shown in Figure 2. We estimate an intrinsic uncertainty in the flux calibration of $\sim 10 \%$. The same data are plotted in Figure 3 as a function of orbital phase. In Figure 4 the 


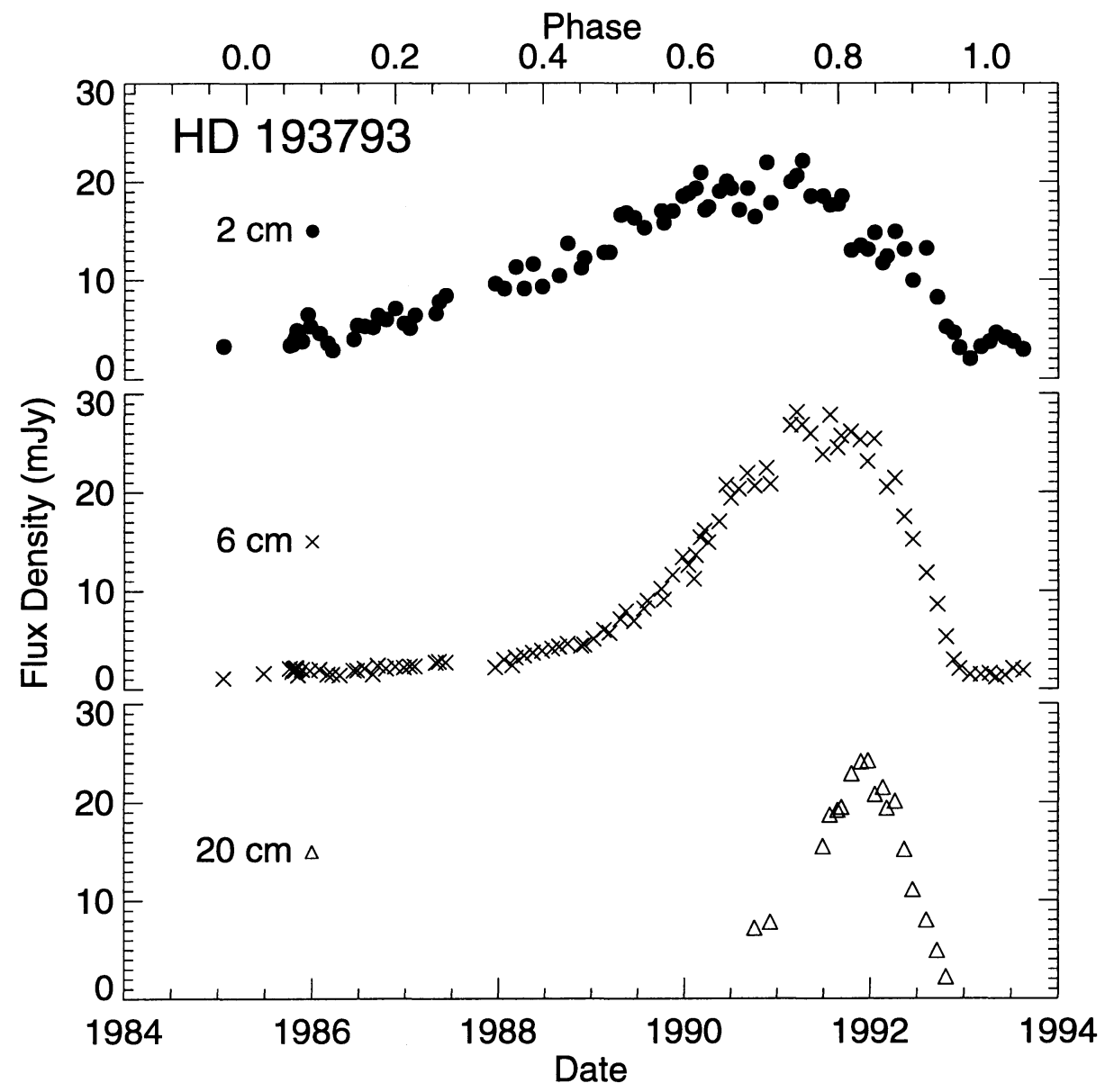

Fig. 2. Radio flux density of HD 193793 at 2,6 , and $20 \mathrm{~cm}$ as determined from our VLA monitoring program. Top axis shows the phase of the orbit, with phase 0 being periastron.

$2 \mathrm{~cm}$ and $6 \mathrm{~cm}$ flux densities are displayed as a shaded surface plot where orbital phase increases in the counterclockwise direction and the vertical height is proportional to the flux density.

\section{Interpretation}

The most striking aspect of the three light-curves is their asymmetry with respect to periastron passage. Of equal interest, there is a strong frequency dependence to the light-curves. The high frequency emission rises earlier, peaks earlier, and begins to decay earlier than emissions at lower frequencies.

At a given frequency, the observed flux density will depend on the intrinsic luminosity of the non-thermal emission as well as the amount of attenuation suffered traversing the binary system. There seems little doubt that both 


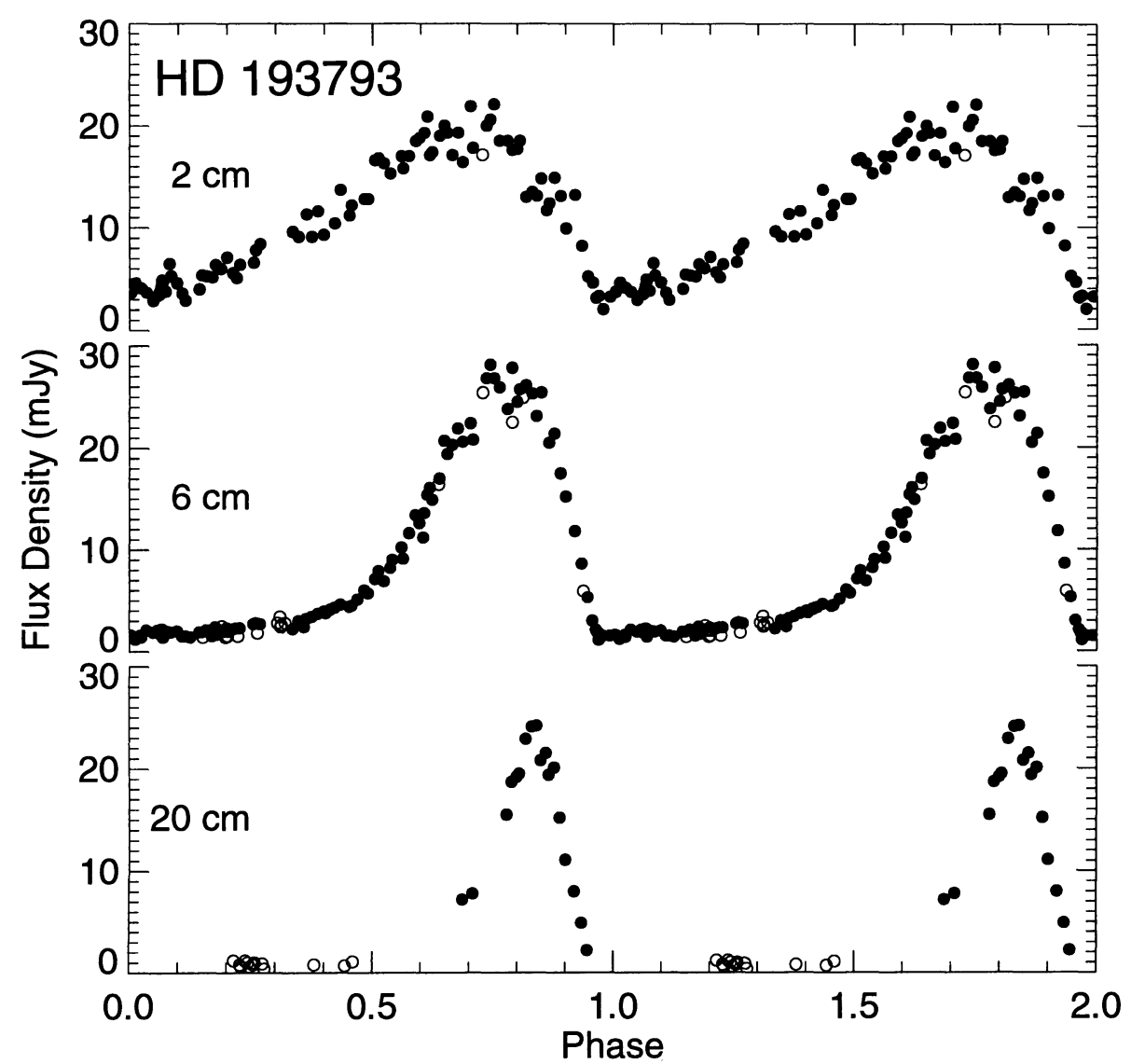

Fig. 3. Radio flux density of HD 193793 at 2,6 , and $20 \mathrm{~cm}$ as a function of orbital phase. Our data are shown with filled symbols, and data drawn from the literature are shown with open symbols. Note the excellent agreement with the other data, which in most cases were not taken during the 1985-1993 cycle. The radio emission repeats almost perfectly from cycle to cycle.

quantities are time-dependent. Consider the scenario in which both stellar winds are spherically symmetric. If attenuation were negligible, the intensity of the interaction should only depend on the separation of the two stars and the light-curve would be symmetric about phase 0.5 (see Williams et al. 1990, Fig. 16). However, strong attenuation will most certainly exist due to free-free absorption in the winds. For example, van der Hucht (1991) estimates the radio photosphere of the WR star's wind to have radii of 20 , 41 , and $101 \mathrm{AU}$ at 2,6 , and $20 \mathrm{~cm}$ respectively. With these large radii one would hardly expect to see radio emission even at $2 \mathrm{~cm}$ when viewing the emission region through the WR wind. Williams et al. (1990) also calculated the light-curve taking attenuation by the WR star's wind into account (his 


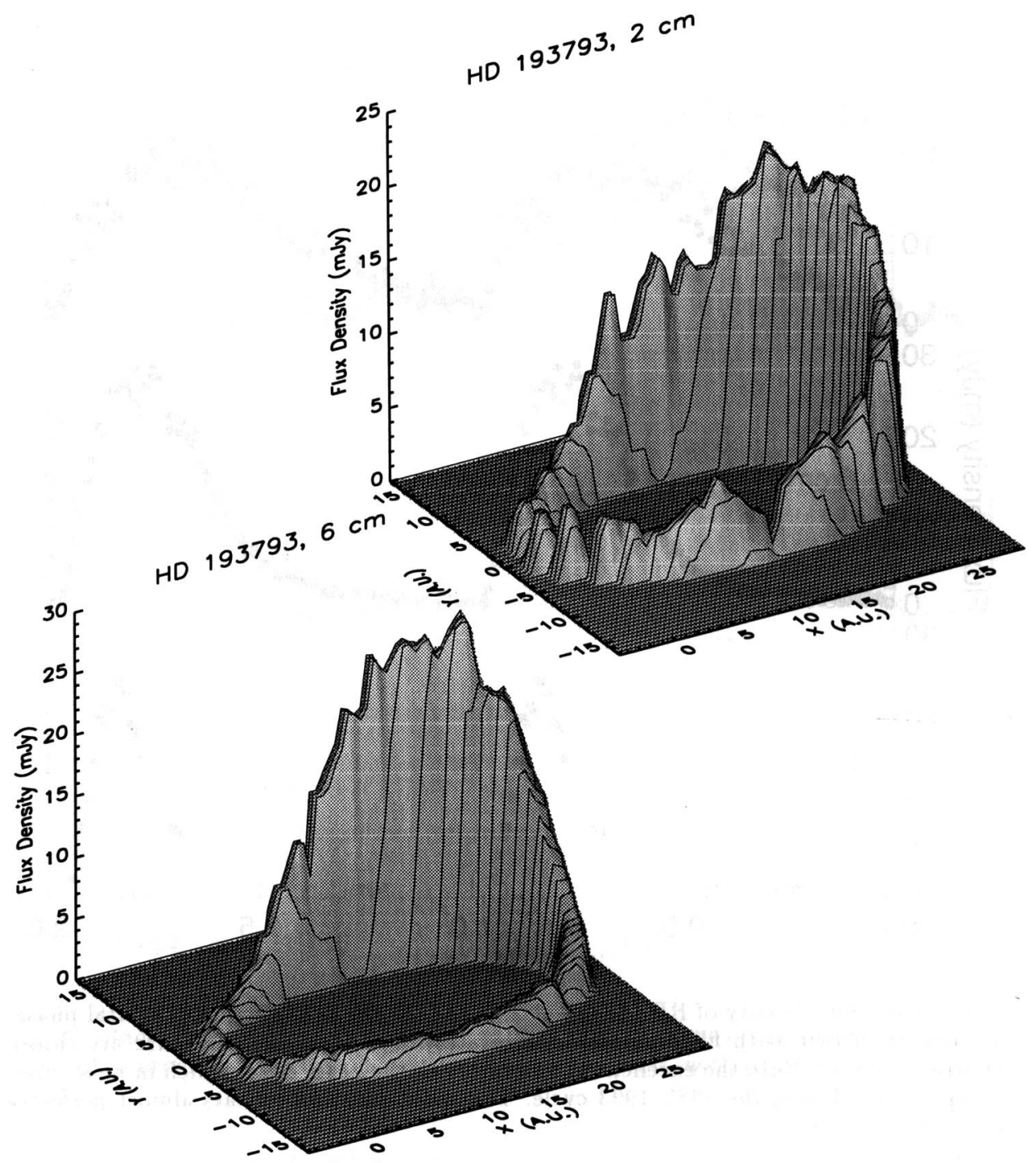

Fig. 4. Radio flux density of HD 193793 at 2 and $6 \mathrm{~cm}$ shown as a function of position in the orbit. Phase increases counterclockwise around the orbit, as shown in Fig. 1.

Fig. 13) but still found poor agreement with observations. In particular, although less symmetric, the derived light-curve still overestimated the radio flux density between phases $0.2-0.5$.

Williams et al. concluded that the light-curve could not be reproduced using a model of spherically symmetric winds and suggested a more complex model which included a hole in the WR star's wind in the $O$ star's shadow. When the hole is oriented along our line of sight the attenuation will be greatly reduced. This model was subsequently elaborated by van der Hucht 

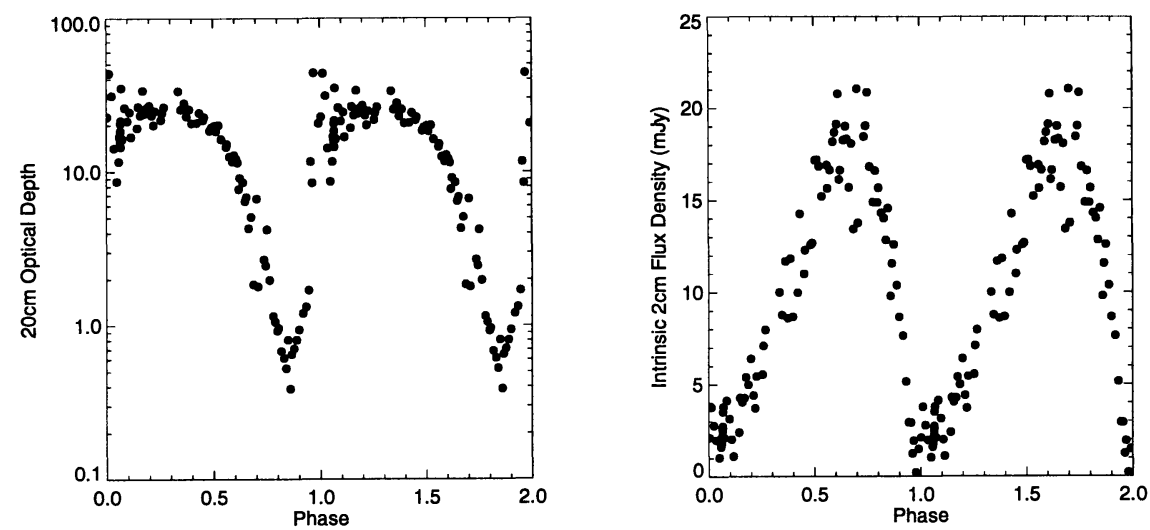

Fig. 5. Optical depth at $20 \mathrm{~cm}$ and intrinsic $2 \mathrm{~cm}$ flux density (non-thermal component only, before attenuation by free-free absorption) as a function of phase. Any radio emission buried below the $2 \mathrm{~cm}$ photosphere is not represented. Derived by fitting 2, 6, and $20 \mathrm{~cm}$ flux densities using a non-thermal spectrum $F_{\nu} \propto \lambda^{0.5}$, free-free absorption with $\tau(\lambda) \propto \lambda^{2.1}$, and a stellar wind spectrum with $F_{\nu}=1 \mathrm{mJy}(\lambda / 6 \mathrm{~cm})^{-0.6}$.

(1991) and Eichler \& Usov (1993). Clearly the radio light-curve predicted by the model will depend strongly on the exact geometry of the shadow cast by the $\mathbf{O}$ star. While this model is appealing in its simplicity and qualitatively would appear to explain the gross shape of the radio light-curves, we find that quantitatively it fails to correctly predict the light-curves shown in Figures 2 and 3.

In particular, we would call attention to the light-curves near phase 0.8 . By the time the $20 \mathrm{~cm}$ flux density peaks at phase 0.85 , the $2 \mathrm{~cm}$ lightcurve has already fallen by nearly $50 \%$ from its peak. In other words, the $2 \mathrm{~cm}$ flux density is falling while the $20 \mathrm{~cm}$ flux density is rising. If the emission mechanism is not changing, i.e., if the spectral index is fixed, then we concluded that from phase 0.7 to 0.85 the intrinsic luminosity is falling at both 2 and $20 \mathrm{~cm}$ but that the attenuation at $20 \mathrm{~cm}$ is falling even faster. If we assume a constant spectral index then we can estimate both the optical depth and intrinsic luminosity as a function of time from the relative flux densities at 2,6 , and $20 \mathrm{~cm}$. Using a spectral index $\alpha=-0.5\left(F_{\nu} \propto \nu^{\alpha}\right)$, the derived attenuation and intrinsic luminosity as a function of phase are shown in Figure 5.

To successfully explain the variation in Figure 5, a model will have to explain a decrease in the intrinsic luminosity after phase 0.7 and a simultaneous sharp drop in the attenuation. These variations are very difficult to accommodate in the context of spherically symmetric winds (SSW). Generally we assume that the shocks which generate the non-thermal radio emission become stronger in the SSW model as the two stars approach each other. Yet the $2 \mathrm{~cm}$ flux density implies that the interaction is decreasing after phase 


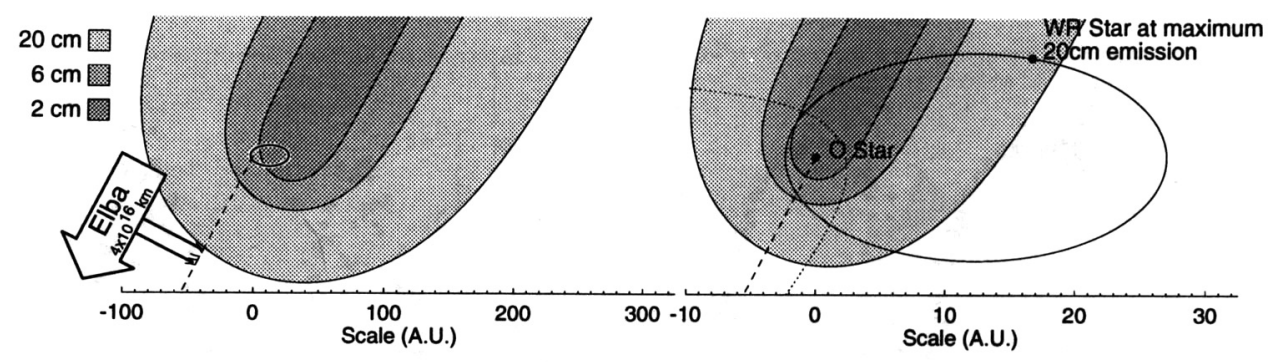

Fig. 6. Free-free absorption by $O$ and WR winds in HD 193793. Orbit and viewing direction are shown. The shaded areas are blocked from our view by wind absorption at 2,6 , and $20 \mathrm{~cm}$. Left: large-scale view showing WR absorption at widest separation. Note that the wind interaction is entirely hidden at $6 \mathrm{~cm}$ and $20 \mathrm{~cm}$. Right: small-scale view showing $\mathrm{O}$ absorption at phase of maximum $20 \mathrm{~cm}$ emission. The position of the shock is shown by the dotted line. Even if there is a hole in the WR wind, the $O$ wind absorption would prevent us from observing much $20 \mathrm{~cm}$ emission.

0.7. Likewise the abrupt drop in the free-free attenuation at phase 0.8 is inconsistent with spherical winds and can only be rationalized in SSW models with a large hole in the WR star's wind caused by a shadow of the 0 star. Such a hole would have to be very wide and have a curved surface to explain the radio light-curves.

In fact, the observation of any $20 \mathrm{~cm}$ emission is not explicable by this model regardless of the size and shape of the hole in the WR wind. Figure 6 shows the regions of the winds that are blocked from our view by free-free absorption in the WR and $O$ winds. If there is a hole in the WR wind then we are looking through the much reduced absorption of the $\mathrm{O}$ wind; however, it is obvious from the figure that the $O$ wind alone has such a large optical depth at $20 \mathrm{~cm}$ that we cannot expect to see a significant amount of $20 \mathrm{~cm}$ emission at phase 0.83 . The only way around this objection is to move the radio emitting region farther from the 0 star so that it is not blocked by the 0 wind. This cannot be accomplished by changing the mass loss rate for the $O$ star, because a larger $\dot{M}$ leads to more $20 \mathrm{~cm}$ absorption and a smaller $\dot{M}$ causes the shock to collapse toward the 0 star.

\section{A New Model}

We suggest that the radio data can be more naturally explained if the WR star's wind is mainly confined to a disk which is tilted with respect to the orbital plane of the stellar system such that the 0 star passes through the disk at phase 0.7. In such a model the interaction of the two winds is strongest when the $\mathbf{O}$ star is immersed in the WR star's wind disk and decreases as the $\mathrm{O}$ star moves away from the disk. The sharp drop in free-free attenuation occurs as the $\mathrm{O}$ star breaks out of the disk, exposing the interaction zone to our line of sight. The problems of absorption by the $\mathrm{O}$ wind are greatly 
reduced because (1) the shocked radio-emitting region does not lie on the line between the two stars and (2) the $O$ wind shock can be farther from the $\mathbf{O}$ star.

The disk model, if correct, will have important ramifications at other wavelengths. In particular, the disk model provides a natural explanation for the sudden formation of dust shortly after periastron (Williams et al. 1990). It is near periastron that the $O$ star enters the WR wind disk for a second time, but in this passage it is much closer to the WR star and sees a correspondingly higher wind density (by a factor of several hundred) over passage at phase 0.7 . The 0 star's introduction into this environment triggers the dust formation. In contrast, in a model assuming spherical winds, it must be considered a remarkable coincidence that periastron passage corresponds to the critical density needed for dust formation.

This new model has striking implications for WR winds. HD 193793 is such a widely separated binary that the wind from the WR star is certainly hardly affected by the presence of the $\mathrm{O}$ star. Presumably all similar WR stars, single or binary, then would also have "disk-winds". Our surprising conclusion, then, is that our radio observations of the unusual binary HD 193793 may indicate the need for sweeping changes in our picture of all WR winds.

\section{Acknowledgements}

We gratefully acknowledge the long-term support of Barry Clark, who without fail scheduled this project on the VLA every month for 8 years. One of us (RHB) would like to thank Mildred Becker for providing travel support for this meeting.

\section{References}

Becker, R.H., White, R.L. 1985, ApJ 297, 649

Eichler, D., Usov, V. 1993, $A p J$ 402, 271

Florkowski, D.R. 1982, in: C.W.H. de Loore \& A.J. Willis (eds.), Wolf-Rayet Stars: Observations, Physics, Evolution, Proc. IAU Symp No. 99 (Dordrecht: Reidel), p. 63

McDonald, J.K., 1947, Pub. DAO 7, 311

Moffat, A.F.J., Lamontagne, R., Williams, P.M., Horn, J., Seggewiss, W. 1987, ApJ 312 , 807

van der Hucht, K.A. 1991, in: K.A. van der Hucht \& B. Hidayat (eds.), Wolf-Rayet Stars and Interactions with other Massive Stars in Galaxies, Proc. IAU Symp No. 143 (Dordrecht: Kluwer), p. 19

Williams, P.M., van der Hucht, K.A., van der Woerd, H., Wamsteker, W.M., Geballe, T.R., Garmany, C.D., Pollock, A.M.T. 1987, in H.J.G.L.M. Lamers \& C.W.H. de Loore (eds.), Instabilities in Luminous Early Type Stars (Dordrecht: Reidel), p. 221

Williams, P.M., van der Hucht, K.A., Pollock, A.M.T., Florkowski, D.R., van der Woerd, H., Wamsteker, W.M. 1990, MNRAS 243, 662 


\section{DISCUSSION:}

Hillier: (a) What is the inclination of the disk, since we would expect significant polarization to occur if there is a disk in this system?

(b) What is the density contrast between the disk and the "normal" wind of the WC star, i.e. what enhancement in density is required to explain the variation around phase $0.7 ?$

Becker: (a) The disk is oriented along the line between the star and phase 0.7. It does not lie in the plane of the orbit, but we do not know its inclination to the orbital plane.

(b) We have not calculated this yet, though we expect that a contrast of 5 to 10 will suffice.

Possibly the $\mathbb{R}$ observations will help determine how dense the WR wind must be in the equatorial phase.

Williams: The scatter at $2 \mathrm{~cm}$ appears to be much greater than that at $6 \mathrm{~cm}$. How much of this is observational and how much is astrophysical?

Becker: To some extent, the increased scatter at $2 \mathrm{~cm}$ will be due to the increased dependence on the weather at $2 \mathrm{~cm}$. But it does appear that the source itself is responsible for some of scatter.

Schmutz: I learned from Walder's 3D hydro calculations (this symposium) that an orbit can create a strong asymmetry in the shock of the wind-wind collision zone. I wonder if it could be possible to explain your data by that kind of model so you wouldn't need a disk.

Becker: Actually WR 140 is such a wide binary that the orbital velocities are quite small compared with the wind velocities, so the asymmetries are modest in this system. In any case, the asymmetry does not appear capable of explaining our observations.

Schulte-Ladbeck: What is the position angle of the disk?

Becker: We do not know the inclination of the disk. Its orientation is along the line joining the WR and $O$ star at phase 0.7 .

Owocki: Do you really mean a "disk" or an asymmetric wind? I suppose you can use the detailed light curve to constrain the density profile, and thus the degree of wind compression.

Becker: Yes, we have in mind an asymmetric wind with a mass loss rate that increases from the pole to the equator. Cassinelli's "WCZ" model is exactly what is required. We plan to do detailed calculations using Cassinelli's models to see how much contrast is required in the wind distribution.

Moffat: The sharp peak at CIII 5696 superposed on the broad WR emission comes from the $\mathrm{O}$-star. This would imply that the $\mathrm{O}$-star is a supergiant or possibly main sequence with a disk around the O-star. Any comments?

Becker: No.

van Kerkwijk: Has the high eccentricity of WR 140 been confirmed? And might this be related to the fact that one apparently needs a system in which the angular momentum vector of the WR star is at a different angle than that of the orbit?

Becker: Williams says it has been repeatedly confirmed that WR 140 has e $\sim 0.85$. The orientation of the disk roughly along the major axis of the orbit does suggest that the eccentricity might be related to the presence of a disk. We do not have a model that explains how the disk could cause the orbit to become highly eccentric, though.

Cherepashchuk: If the disk exists around the WR star it is most probable that it is a windcompressed disk like that described by Joe Cassinelli. If there is the misalignment between rotational axes of WR star and orbital rotational moment long-periodic precession of the rotational axis of WR star may exist. This leads to long-term radio variability from one orbital period to another. It could be interesting to observe this effect. 
Becker: The separation is so large in this system that I expect this effect would be very small during one orbital pass. Still, it is an interesting suggestion.

Pollock: 1. Is it the case that the luminosity vs binary phase variation you derive is based on the assumption that the $2 \mathrm{~cm}$ emission is optically thin? Are you confident that this is the case? 2. Why do you think that the X-ray luminosity continues to increase up to at least $\phi=0.89$ from the ROSAT data?

Becker: 1. We assume that the nonthermal radio spectrum is a power law. A fit to the data then requires that the system is optically thin at $2 \mathrm{~cm}$ wavelength. 2 . I think that the X-ray light curve is too uncertain to draw firm conclusions from.

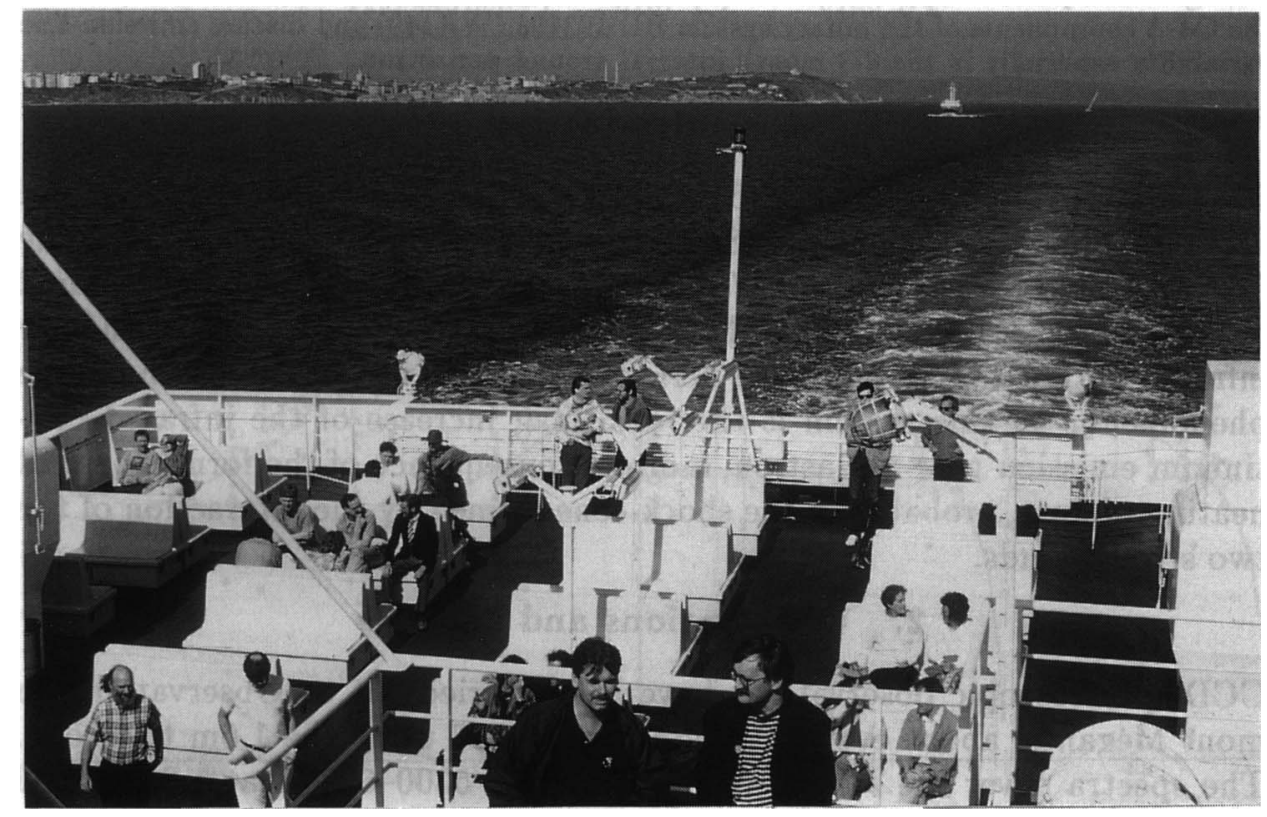

Corchoran, Pollock 